\title{
PRENATAL FORMATION OF CORTICAL INPUT AND DEVELOPMENT OF CYTOARCHITECTONIC COMPARTMENTS IN THE NEOSTRIATUM OF THE RHESUS MONKEY ${ }^{1}$
}

\author{
PATRICIA S. GOLDMAN-RAKIC
}

Section of Neuroanatomy, Yale University School of Medicine, New Haven, Connecticut 06510

\begin{abstract}
The timing, hemispheric laterality, and mode of termination of input from the prefrontal association cortex to the neostriatum were studied in fetal and neonatal rhesus monkeys using autoradiography for tracing connections. In addition, the cytological maturation of the neostriatum was examined in Nissl-stained sections from the same and other monkeys of selected prenatal and postnatal ages.

A small contingent of corticostriatal axons reaches both the caudate nucleus and the putamen by the 69th embryonic day (E69) of the 165-day gestation period in this species and steadily expands over the next 3 fetal months. Throughout this period, ipsilateral prefrontostriatal connections predominate, and only a small and variable amount of label is detectable over the contralateral neostriatum. A major feature of the developing corticostriatal projection is a transfiguration in the distribution of its terminals: from E69 to E95, cortical terminals are distributed uniformly among neostriatal neurons; beginning around E105, areas of higher and lower grain density begin to emerge until finally, by E133, 250- to 500- $\mu \mathrm{m}$-wide circular and elliptically shaped label-free cores perforate a field of densely labeled cortical terminals as in the neostriatum of the adult monkey (Goldman, $P$. S., and W. J. H. Nauta (1977) J. Comp. Neurol. 171: 369-386).

The cytoarchitectonic composition of the neostriatum also changes during gestation: from E69 through E95, the small postmitotic neurons of the immature neostriatum are packed densely and, for the most part, are distributed homogeneously; by E105, they become segregated into cellular islands consisting of densely packed neurons that are encapsulated by fiber-rich annuli and embedded in a matrix of less densely arrayed neurons. The shape and size of the islands in Nissl-stained sections correspond to label-free cores in autoradiograms of fetuses with cortical injections, while the surrounding annuli and adjacent matrix cells correspond to areas of dense accumulation of label. Thus, the formation of the corticostriatal projection in primates involves a transformation in the distribution of ingrowing terminals synchronized with changes in cellular organization of the neostriatum.
\end{abstract}

The corticostriatal projection is one of the major efferent pathways of the frontal association cortex in primates (Kemp and Powell, 1970; Goldman and Nauta, 1977). Recent autoradiographic studies conducted on the rhesus monkey have demonstrated that this important class of prefrontal efferents is already well developed at birth when it exhibits an intricate, fenestrated pattern characteristic of the distribution of corticostriatal terminals in mature monkeys (Goldman and Nauta, 1977; Goldman-Rakic, 1981). Therefore, data on the timing, sequence, and mode of development of corticostriatal connections in this species can be obtained only by prenatal

\footnotetext{
' This work was supported by United States Public Health Service Grants NS 16666 and MH 00298.
}

studies. Recent advances in neurosurgical manipulation of the primate fetus (Taub et al., 1974; Rakic, 1976; Goldman and Galkin, 1978) and the refinement of neuroanatomical tracing techniques (Cowan et al., 1972) have made it possible to obtain such information.

The present investigation was designed to address several basic developmental questions. The first goal was to determine the timetable for the formation of the corticostriatal projection in a primate-information which is still not available for any mammalian species. Secondly, we examined the laterality of this projection at formative stages in fetuses. Although, in adult monkeys, prefrontostriatal connections are predominantly ipsilateral (Goldman and Nauta, 1977; Goldman, 1978; Künzle, 1978; Yeterian and Van Hoesen, 1978), recent findings of aberrant contralateral corticostriatal connec- 
tions following prenatal lesions of the prefrontal cortex have raised the possibility that, in fetuses, corticostriatal projections may be bilateral (Goldman, 1978). Thirdly, we analyzed the mode of development of the intricate pattern of corticostriatal terminal fields with the aim of determining whether this input initially overlaps with other neostriatal input before becoming segregated in a manner similar to the biphasic development of afferent territories in visual (Rakic, 1976; Hubel et al., 1977) and other sensory (Price et al., 1976) systems. Finally, we examined the correlation between the development of terminal fields and the maturation of the cytoarchitectonically distinct cellular compartments that have been observed recently in the neostriatum of the mature primate (P. S. Goldman-Rakic, submitted for publication).

\section{Materials and Methods}

Subjects. Ten monkeys were injected with isotopes at embryonic (E) ages E69, E86, E95, E104, E120, E123, F124, F133, F151, and E152 and were sacrificed 1 to 3 days later. Four monkeys were injected postnatally (P) at P3, P4, P5, and P69 and were sacrificed after 2 to 7 days. In addition, a number of unoperated monkeys of selected fetal or postnatal ages were used for cytological analyses. The brains of the latter animals were embedded in celloidin, cut in the frontal or horizontal planes, and stained with cresyl violet.

Prenatal injections. Following laparotomy of timed pregnant monkeys, the fetal head was exposed through an incision in the uterus (Goldman and Galkin, 1978). With the head propped by a surgical assistant, the scalp, periosteal tissues, and calvarium were opened in successive steps and the dura was incised over the prospective dorsolateral prefrontal cortex. To provide control of injection depth, we used a stereotaxic carrier attached to a Siegler Flexbar Holder/Positioner. A 5- or 10- $\mu \mathrm{l} \mathrm{Ham-}$ ilton microsyringe with a 26 gauge needle was attached to the electrode carrier and positioned about the target region.

Although primary fissures in fetal rhesus monkeys do not develop fully until the last third of gestation, the principal sulcus can be visualized as a slight indentation or dimple by E95. In fetuses in which this landmark was apparent, the injection was placed in the middle of the developing dorsal bank. In younger fetuses, in which the anlage of the principal sulcus could not be identified, the injections were placed in the center of the anterodorsal part of the frontal lobe. The needle was aimed perpendicularly or at a slight angle to the pial surface and lowered to an intracerebral depth of 1 to $1.5 \mathrm{~mm}$. A mixture of equal parts $\left[{ }^{3} \mathrm{H}\right]$ leucine and $\left[{ }^{3} \mathrm{H}\right]$ proline $(100$ $\mu \mathrm{Ci} / \mu \mathrm{l})$ was injected intracortically in volumes ranging from 0.33 to $1.00 \mu \mathrm{l}$ of saline. Following the injections, the dura was sutured, the cranial defects were filled with Gelfoam, and the fetal skin was closed in one layer. The fetus was replaced in utero and the mother was given prophylatic doses of penicillin and a smooth muscle relaxant, Vasodilan (Mead Johnson).

Perfusion. One to 7 days following the intracerebral injections, the fetus was delivered by cesarean section and its umbilical cord was clamped and severed. Neonates were anesthetized with sodium pentobarbital (40 $\mathrm{mg} / \mathrm{kg}$ ) before intracardial perfusion with $10 \%$ formal saline. The brain was removed from the cranial vault, photographed at different angles, and postfixed in formalin for an additional 1 to 2 weeks before being cut and processed for autoradiography.

Autoradiography. The entire brain was prepared for frozen sectioning as three equal sized coronal blocks. After saturation by $30 \%$ sucrose/formalin, the blocks were embedded in gelatin/albumin to facilitate sectioning and, in particular, mounting of the delicate fetal tissue of a large brain (Crane and Goldman, 1979). Sections, 25 to $35 \mu \mathrm{m}$ thick, were cut on a freezing microtome, and, depending on the size of the brain, every second or every fifth section was mounted on chrome-alum gelatincoated slides and then dipped with Kodak NTB-2 nuclear track emulsion. After exposure from 10 to 16 weeks, the autoradiograms were developed in Kodak D-19 and counterstained with cresyl violet or thionin.

Injection sites. The location and extent of the injection sites were mapped postmortem using microscopic examination of serial sections. In addition, corticothalamic projections and other subcortical terminations were used as a further source of data relevant to defining the injection site. Only cases in which the radioactive amino acids were well confined to the prefrontal cortex, as shown in Figure 1, are included in this study.

\section{Results}

Timing and mode of development of cortical input and striatal compartments. The youngest fetus was injected at E69 and sacrificed at E70 (E69-E70). At this early stage of gestation, the primate frontal lobe does not show involutions into sulci and gyri (Fig. 1 $A$ ). Although, at E69, the cortical plate is already packed densely with darkly stained neurons, these cells are not organized yet into horizontal laminae visible in Nissl-stained sections. Prospective layer 2 and part of layer 3 are not generated yet (P. Rakic and G. Wikmark, unpublished $\left[{ }^{3} \mathrm{H}\right]$ thymidine observations) and cells in the deep layers form a thin sheet beneath the marginal zone of the cerebral vesicles.

In the E69-E70 fetus, the neostriatum is a small structure situated beneath the ganglionic eminence containing numerous tightly packed and uniformly distributed immature neurons (Fig. 3A). At this age, the anterior limb of the internal capsule which penetrates the neostriatum contained heavily labeled fibers that originate in the prefrontal cortex and descend to the thalamus and to various levels of the brainstem. However, only a fine spray of silver grains was observed over the developing neostriatum and then only over the ipsilateral hemisphere (Fig. 2A). Grains were distributed uniformly in the dorsolateral margin of the caudate and in the mediodorsal sectors of the putamen; there was no indication of the type of clustering of labeled fibers that is characteristic of the corticostriatal pathway in the postnatal animal (Goldman and Nauta, 1977).

The density of radioactive grains increased over the caudate nucleus and putamen in fetuses injected at E85 and E95, but again only in the ipsilateral hemisphere (Fig. 1B). At these fetal stages in the rhesus monkey, all neostriatal neurons have been generated, but many postmitotic cells probably still have not completed the migrations from their site of origin in the nearby ganglionic 


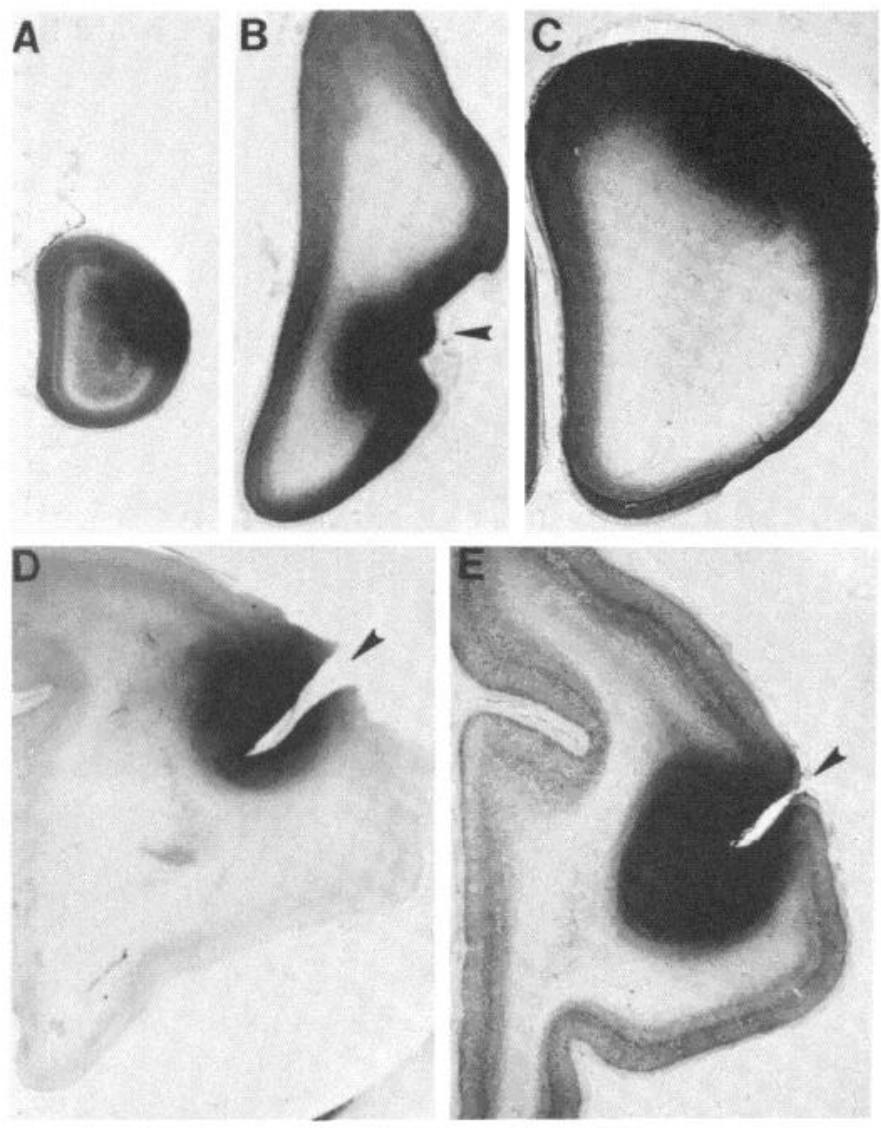

Figure 1. Coronal sections through injection sites in the frontal lobes of five rhesus monkey fetuses of various embryonic (E) ages. Each fetus was exteriorized from the uterus and its cerebrum was exposed and injected with $\left[{ }^{3} \mathrm{H}\right]$ leucine and $\left[{ }^{3} \mathrm{H}\right]-$ proline. Following injection, the fetus was returned to the uterus and sacrificed 1 to 3 days later. The days of injection, followed by the day of sacrifice, are: $A, \mathrm{E} 69-70 ; B, \mathrm{E} 95-96 ; C$, E104-105; $D$, E123-125; E, E152-155. To display the maximal extent of the spread of radioactivity at the injection site, each autoradiogram was exposed at least 10 weeks. The principal sulcus or its anlage if present in the section is indicated by a black arrow. Magnification $\times 5.5$.

eminence to their final locations in the neostriatum (Brand and Rakic, 1979a). Through E95, both neostriatal nuclei are still densely packed and relatively homogeneous cellular structures, but some cytoarchitectonic complexity can be detected (Fig. $3, B$ and $C$ ). In the injected hemisphere, the fibers that originate in the prefrontal cortex again were localized primarily in the dorsalmost half of the ipsilateral caudate nucleus and putamen. As in the fetus injected at E69, silver grains in fetuses injected at E85 and E95 were distributed uniformly within the neostriatum (Fig. $2 B$ ).

The first indication of a patterned corticostriatal projection was discernible in a fetus that was injected at E104 and sacrificed at E105. At this stage, terminals of prefrontal axons were still distributed rather uniformly, but some evidence of segregation of afferent input began to emerge in the form of areas of higher and lower grain density within an otherwise homogeneous field of label (Figs. $2 C$ and $4 A$ ). At the same time, remarkable changes were apparent in the cytoarchitectonic appearance of the neostriatum. Examination of Nissl-stained histological sections revealed the emergence of irregularly shaped aggregates of neurons surrounded by cell-free rings or perimeters. The cytoarchitectonic complexes thus formed can be described as cellular islands embedded in a less defined matrix of neostriatal cells (Fig. 5, $A$ to $E$ ). These islands, many of which appeared oval or elliptical in coronal section, showed considerable variation in their long axis diameters. Islands ranging in width from 200 to $500 \mu \mathrm{m}$ were especially prominent in $40-\mu \mathrm{m}$ celloidinembedded tissue (Fig. 5, $D$ and $E$ ) but also were visible in 25- to $35-\mu \mathrm{m}$ frozen sections prepared for autoradiography (Fig. 5, $A$ to $C$ ). A related observation in the counterstained autoradiograms was that a number of the cell-poor zones that encapsulated the islands contained a higher concentration of labeled corticostriatal fibers, while cellular islands at this age were less radioactive (Fig. 6, $A$ to $D$ ). Discontinuities in the distribution of radioactivity became even more prominent in the brains of three older fetuses injected between E120 and E124 (Fig. 2D).

The fully adult-like pattern of a prefrontal terminal matrix which surrounds oval or elliptical territories that are virtually devoid of such input was first fully developed in a fetus injected at E133 and sacrificed at E134 (Figs. $2 E, 4 B$, and $7, A$ and $B$ ). It is noteworthy that the crosssectional diameters of the projection-free cores or islands surrounded by a field of labeled prefrontal fibers measure 250 to $500 \mu \mathrm{m}$ at this developmental stage and thus are equivalent to or only slightly smaller than the long axis diameters of similar islands in mature neostriatum which measure approximately 300 to $600 \mu \mathrm{m}$ (Bugbee and Goldman-Rakic, 1980) (Figs. 7, $C$ and $D$ and $8, A$ to $D$ ). The same fenestrated pattern of corticostriatal terminals was observed in two older fetuses, one injected at E151 and sacrificed at E152 and the other injected at E152 and sacrificed at E155. At these late fetal and also at early postnatal stages of development, labeled cortical axons are confined to the cell-free annuli and matrix zones of the neostriatum and do not appear in the territories occupied by the island cells. This selective pattern of connectivity is illustrated in a postnatal monkey in Figure $7, A$ to $D$. A dark-field photograph of the caudate nucleus in this case displays the labeled prefrontal fibers surrounding almond-shaped core territories devoid of labeled fibers (Fig. $7 A$ ). The very same section viewed under bright-field illumination (Fig. 7, $B$ to $D$ ) reveals that the label-free cores contain cellular islands. Although these cytoarchitectonic compartments are best differentiated in the postnatal monkey, at the same time, they are easier to visualize in fetal monkeys perhaps because of the high packing density of cells in the immature brain.

As far as laterality of corticostriatal projections at these later gestational ages is concerned, evidence for a contralateral innervation of the neostriatum was observed for the first time in fetuses injected between E105 and E124 and was most evident in the fetus injected at E133 (Fig. 4B). The contralateral fibers were distributed in a location that was mirror symmetric to the location of prefrontal projections in the ipsilateral neostriatum. However, the label present in the contralateral neostriatum, even in the E133 fetus, was far less dense and less extensive than the ipsilateral counterpart in the same 

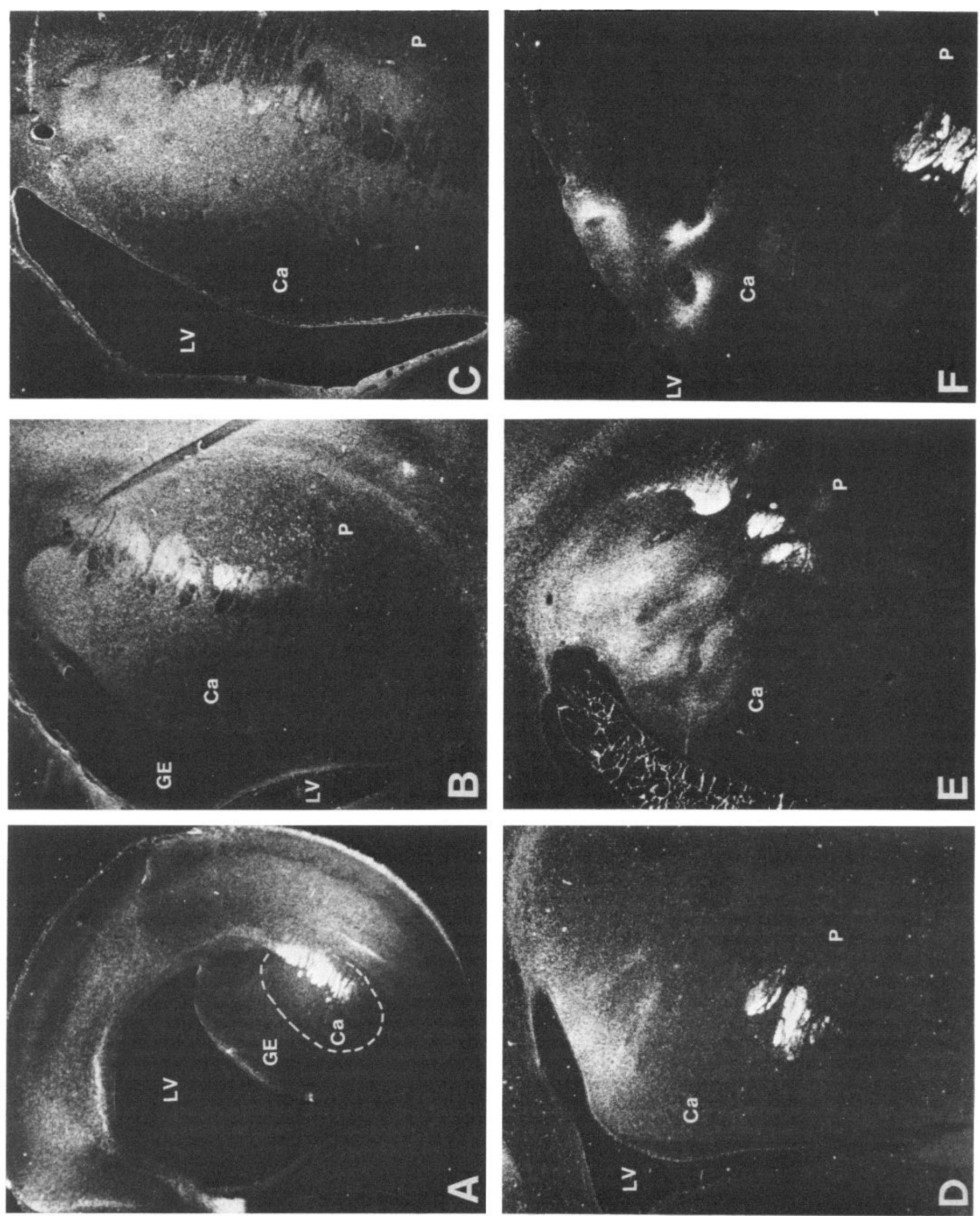

a.

。 
sections. Fetuses injected after E133, as well as postnatally, all exhibited heavy concentrations of label in the caudate nucleus and putamen of the ipsilateral hemisphere, whereas label in the same structures in the contralateral hemisphere was extremely sparse and barely above background (Fig. $4 C$ ).

\section{Discussion}

Timetable of corticostriatal innervation. The present study provides novel information on the timing, topography, and mode of distribution of corticostriatal fibers in the developing primate brain. This major class of corticostriatal efferents reaches its subjacent ipsilateral target by E69, well before the middle of gestation in this species and well before the emergence of cellular differentiation in the neostriatum. The establishment of a connection between the frontal cortex and the neostriatum by E69 or earlier is in harmony with $\left[{ }^{3} \mathrm{H}\right]$ thymidine evidence obtained in the same species that neurons in cortical layer 5 which give rise to the corticostriatal projection are generated some time between E45 and E63 (P. Rakic and G. Wikmark, unpublished observations). Thus, at least the earlier generated layer 5 neurons have had ample time to migrate to the cortex and to elaborate efferent axons that reach the nearby neostriatum. Our findings are also in general agreement with the timing of development of efferent subcortical connections from the primary visual cortex in the same species (Shatz and Rakic, 1981). According to this study, corticotectal and corticogeniculate input was present for the first time in a fetus injected at E69. It is probably no coincidence that the corticostriatal projections described here develop around the same time. Although a few prefrontal components of the corticostriatal projection may reach their target even earlier than E69, the major influx of corticostriatal fibers occurs during the midgestational period and is paralleled during this period by a dramatic increase in the density of synapses in the neostriatum (Brand and Rakic, 1979b). On the basis of the autoradiographic data obtained in the present study, it is likely that a large proportion of these synapses are formed by axons originating from the neocortex.

Hemispheric laterality of corticostriatal projections. There appears to be considerable regional variation in the extent to which various cortical regions in adult mammals issue projections to the contralateral neostriatum. Thus, the motor cortex seems to project to the neostriatum bilaterally in both primates (Künzle, 1975) and carnivores (Cospito et al., 1980; Tanaka et al., 1980) although the contralateral component is usually less dense than the ipsilateral. In contrast, the somatosensory cortical input to the striatum is predominantly, if not exclusively, ipsilateral (Jones et al., 1977). The projection from prefrontal cortex to neostriatum is also primarily ipsilateral although a minor contralateral component of the corticostriatal pathway has been noted (Goldman, 1978; Fallon and Ziegler, 1979).

The regional differences in laterality of corticostriatal projections raise the question of whether these differences exist from the earliest stages of ontogeny or whether all cortical areas are connected initially with the neostriatum bilaterally and some lose their contralateral component at later stages as has been demonstrated, for example, for retinotectal fibers in the developing rat brain (Land and Lund, 1979). A selective elimination of crossed connections might explain the enhanced contralateral corticostriatal projections that are observed after unilateral prefrontal ablation in the prenatal period (Goldman, 1978) and would be similar to the mechanism proposed for the development of the regionally selective distribution of callosal fibers in the feline occipital lobes (Innocenti et al., 1977). However, the present study revealed no indication that the crossed innervation was more prominent at earlier than at later stages of gestation. In the youngest fetuses, injected between E69 and E95, it was difficult even to determine whether grain concentrations in the contralateral neostriatum exceeded background or whether, instead, fibers from the prefrontal cortex had failed to reach the contralateral cortex at these ages. In fetuses injected at later fetal ages, as well as in postnatal monkeys, grain densities over the contralateral neostriatum were variable yet generally only slightly above background in the mirror image location to the prefrontostriatal input of the ipsilateral hemisphere. Inexplicably, the highest concentration of grains in the contralateral neostriatum was observed in one fetus that was injected at E133 and sacrificed 1 day later. Perhaps some of the crossed corticostriatal input observed in these as well as in other studies (Fallon and Ziegler, 1979) is a consequence of transneuronal transport through neurons in the contralateral cortex to which the prefrontal cortex also projects (Goldman-Rakic, 1981). Although contralateral corticostriatal projections can be demonstrated in monkeys with unilateral prefrontal ablations, these findings would not necessarily preclude the transneuronal route in normal monkeys. It should be emphasized that neither in the fetus injected at E133 nor in any other did the contralateral label approach in density or in extent that observed in the ipsilateral neostriatum. The lack or sparseness of label over the contralateral neostriatum cannot be attributed to limitations of the autoradiographic method since, in the very same sections, heavy labeling was present in the corpus callosum and in the contralateral prefrontal cortex (Goldman-Rakic, 1981). Failure to detect significant quantities of label in the contralateral neostriatum emphasizes that

Figure 2. ${ }^{2}$ Dark-field illumination photographs of autoradiograms prepared from coronal sections through the neostriatum of fetal monkeys that were sacrificed 1 to 3 days after they received injections of $\left[{ }^{3} \mathrm{H}\right]$ leucine and $\left[{ }^{3} \mathrm{H}\right]$ proline into the developing dorsolateral prefrontal cortex. The days of injection, followed by the day of sacrifice, are: $A, \mathrm{E} 69-70 ; B, \mathrm{E} 86-87 ; C, \mathrm{E} 104-105 ; D$, E120-123; E, E133-134; F, P4-11. Dashed white lines in $A$ outline the boundary of neostriatum which, at this early stage, is overshadowed by the large proliferative center, the ganglionic eminence ( $G E$ ). Readings from $A$ to $F$ in this figure display the progressive compartmentalization of corticostriatal terminal fields from diffuse and uniform to fenestrated. Magnification $\times 10$.

\footnotetext{
${ }^{2}$ The abbreviations used on the figures are: Ca, caudate nucleus; CC, corpus callosum; GE, ganglionic eminence; IC, internal capsule; LV, lateral ventricle; $P$, putamen.
} 

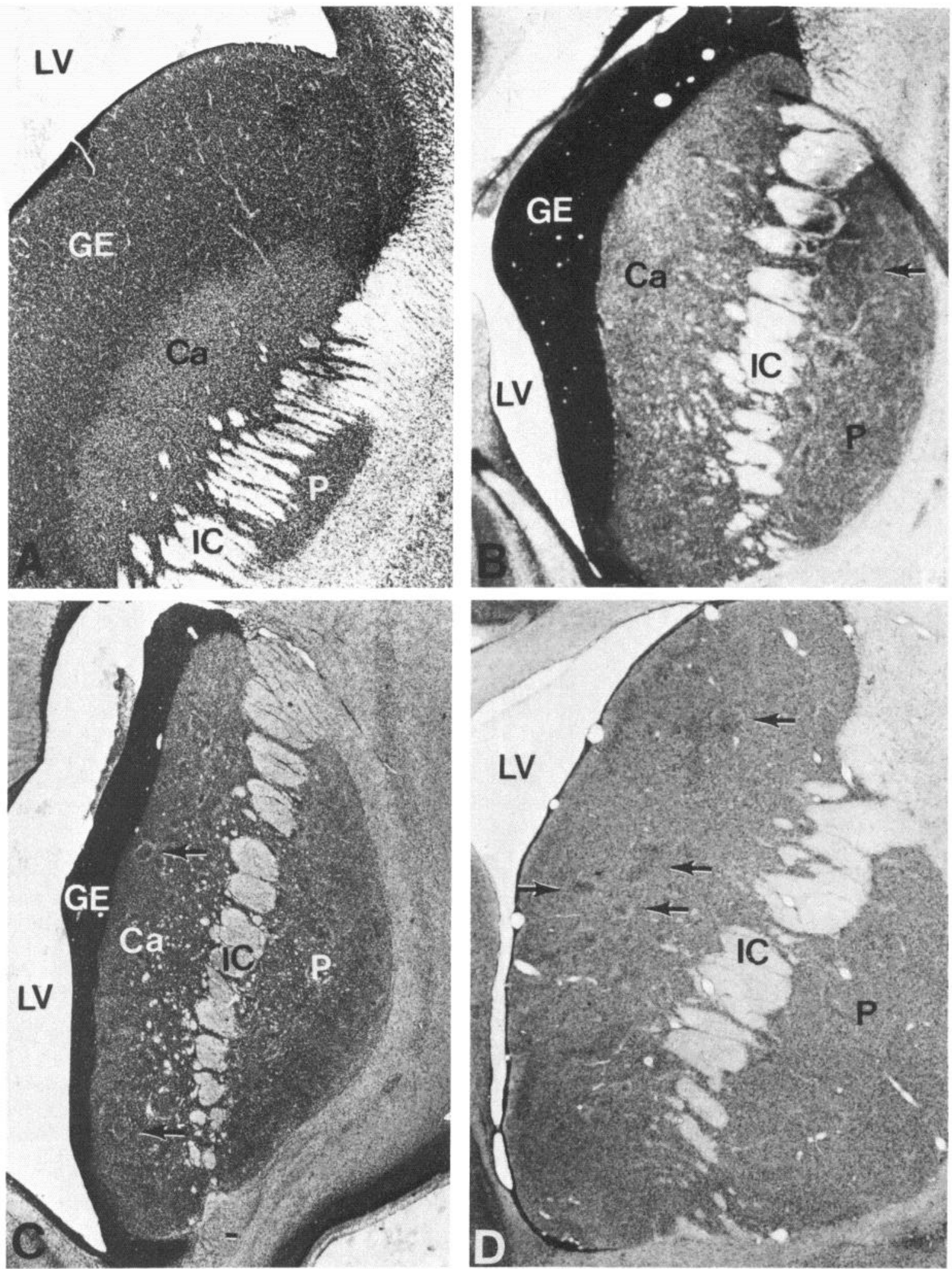

Figure 3. Cresyl violet-stained coronal sections cut across the developing neostriatum depicting cellular organization at different fetal or postnatal ages. $A$, At E70, the neostriatum is composed of immature, densely packed, and uniformly distributed neurons; $B$ and $C$, cellular organization is still basically homogeneous, but some cellular islands begin to emerge in the neostriatum between E87 and E96 (arrows); D, cellular islands are present and visible in a 2-month-old monkey. Magnification of the photographs decreases with the age of the specimen to permit reproduction in a combined format: $A, \times 23 ; B, \times 12 ; C, \times 10 ; D, \times 7.7$. 

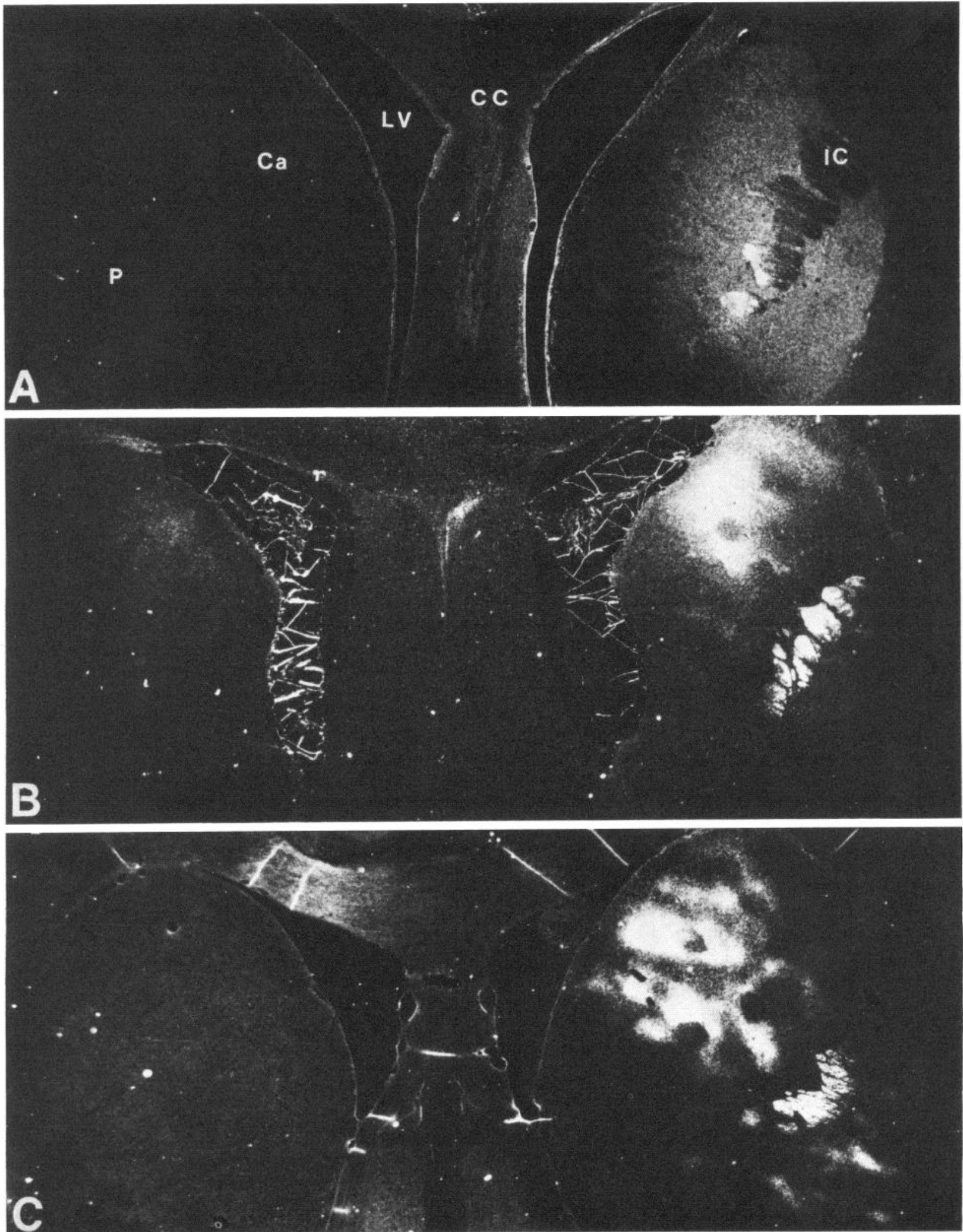

Figure 4. Dark-field illumination photographs of the neostriatum in both hemispheres of monkeys whose prefrontal cortex was injected at E104 $(A), \mathrm{E} 133(B)$, and $\mathrm{P} 4(C)$. The injected hemisphere is shown on the right. Label could not be detected in the contralateral hemisphere of fetuses injected earlier than E104 and was barely above background in the fetus injected at E104. A meager contralateral projection can be seen in the left caudate nucleus in the E133 fetus, but the crossed innervation is much less dense and extensive than the uncrossed ipsilateral projection. Magnification $\times 10$. 

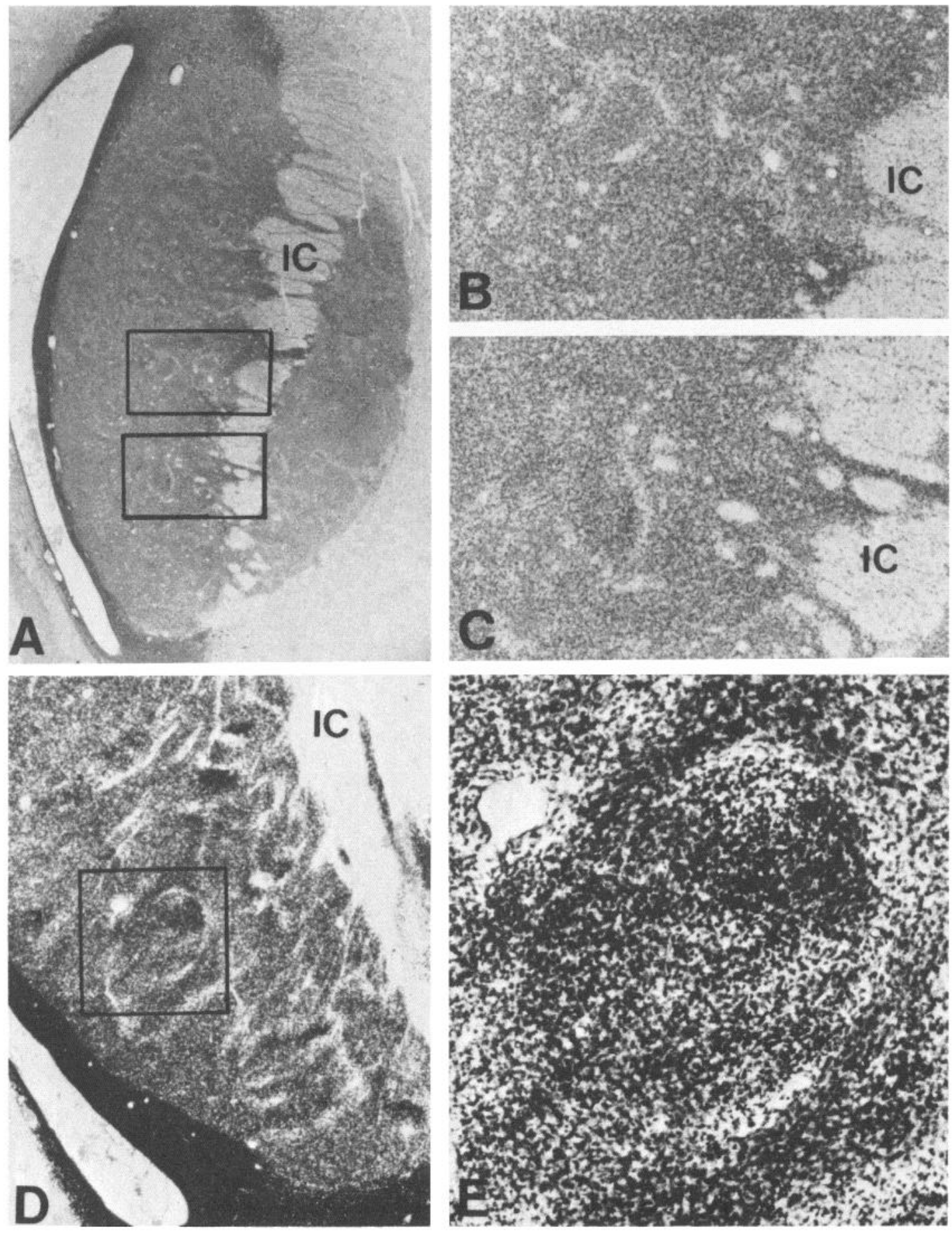

Figure 5. Cellular islands displayed in bright-field illumination photographs of Nissl-stained sections cut through the neostriatum of two fetal rhesus monkeys sacrificed at E105. $A, 35-\mu \mathrm{m}$ Nissl-stained frozen section from the fetus shown also in Figures $1 C, 2 C$, and $4 A$ whose prefrontal cortex was injected with isotopes 1 day earlier. Magnification $\times 12$. $B$ and $C$, Higher magnification photographs of the portion of the field shown in the rectangular insets in $A$. The cellular islands at this stage already measure 200 to $500 \mu \mathrm{m}$ in cross-sectional diameter (long axis). Magnification $\times 52.5$. $D$, A 35- $\mu \mathrm{m}$ Nissl-stained celloidinembedded section from another fetal brain of the same age cut in horizontal section showing the formation of irregularly shaped cellular islands in this plane. Magnification $\times 49$. E. Higher magnification view of the island encompassed by the rectangle in $D$ reveals the globoid shape of an island along its longitudinal axis and the cell-free annulus that separates it from the surrounding matrix zone. Magnification $\times 160$.

the crossed input from prefrontal cortex must be negligible, if present at all, and makes it unlikely that selective elimination could account for regional differences in the laterality of corticostriatal connections in primates. Fur- thermore, the present results indicate that enhancement of the contralateral component in monkeys with unilateral removal of the prefrontal cortex prenatally (Goldman, 1978) probably represents an expansion of the ter- 

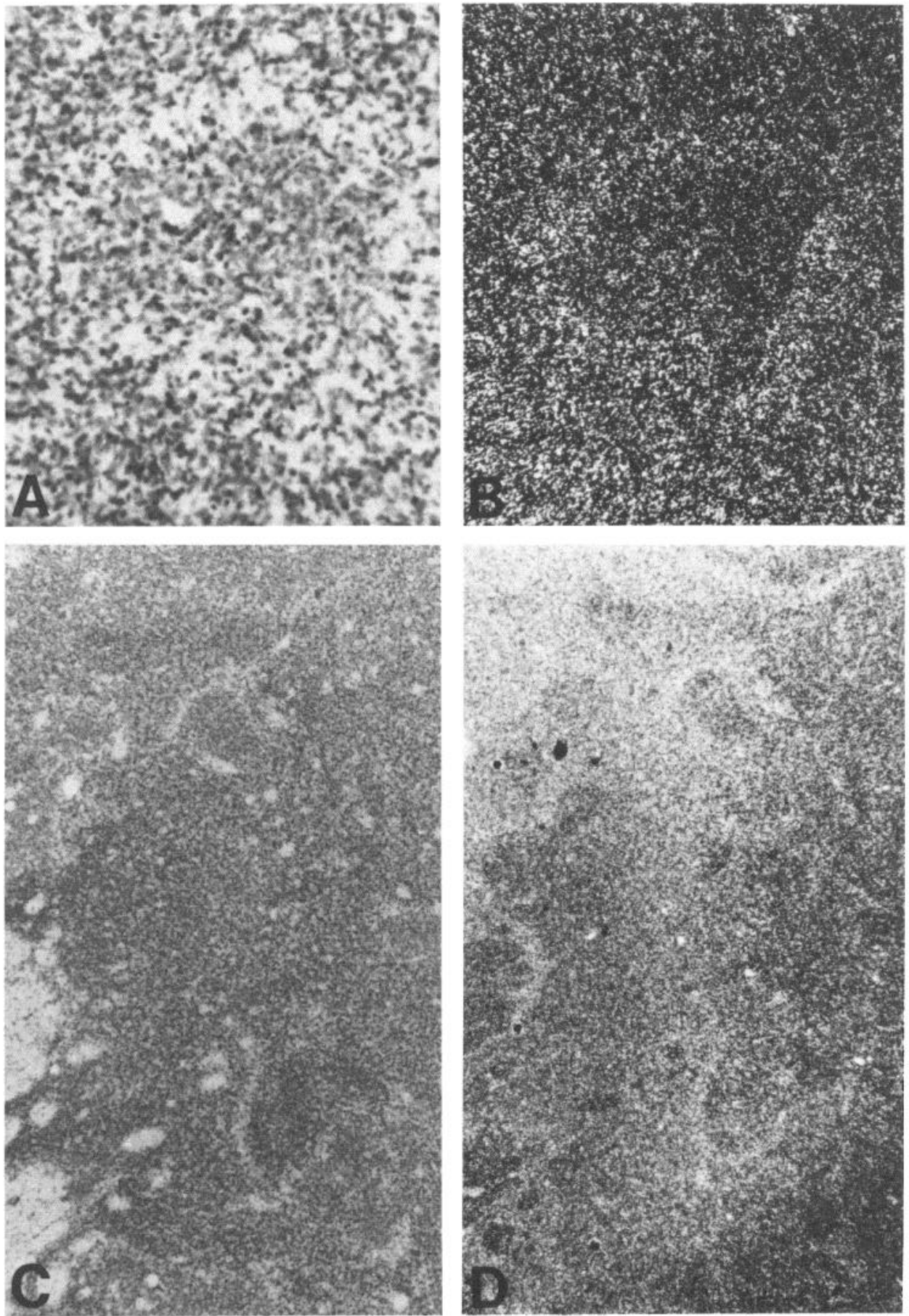

Figure 6. Bright-field and dark-field photographs of cellular islands observed in a fetus injected at E104 and sacrificed 1 day later. $A$, An example of a cellular island observed in a Nissl-stained section through the caudate nucleus near the lateral ventricle; $B$, dark-field photograph of the very same section showing accumulation of radioactive label in the cell-poor perimeter around the island; $C$, other examples of islands shown in a Nissl-stained section under bright-field illumination at lower power; $D$, autoradiogram of the same section displaying higher accumulations of radioactivity in the cell-poor perimeters and surrounding matrix zones than over the cellular islands at this fetal age.

minal fields of the normally sparse crossed cortical efferents rather than a simple failure of fiber retraction.

Development of the segregated pattern of corticostriatal innervation. A finding that may have considerable bearing on our understanding of synaptic connectivity in the neostriatum is that the prenatal genesis of the corticostriatal innervation involves a transformation from a diffuse pattern of termination to one that is intricately patterned. As shown in the summary diagram (Fig. 9), this rearrangement of connections is accomplished during midgestation in two well defined stages of development. The first phase in which the corticostriatal terminals are distributed uniformly lasts from approximately E69 to E95. In the second stage, between E104 and E133, areas 

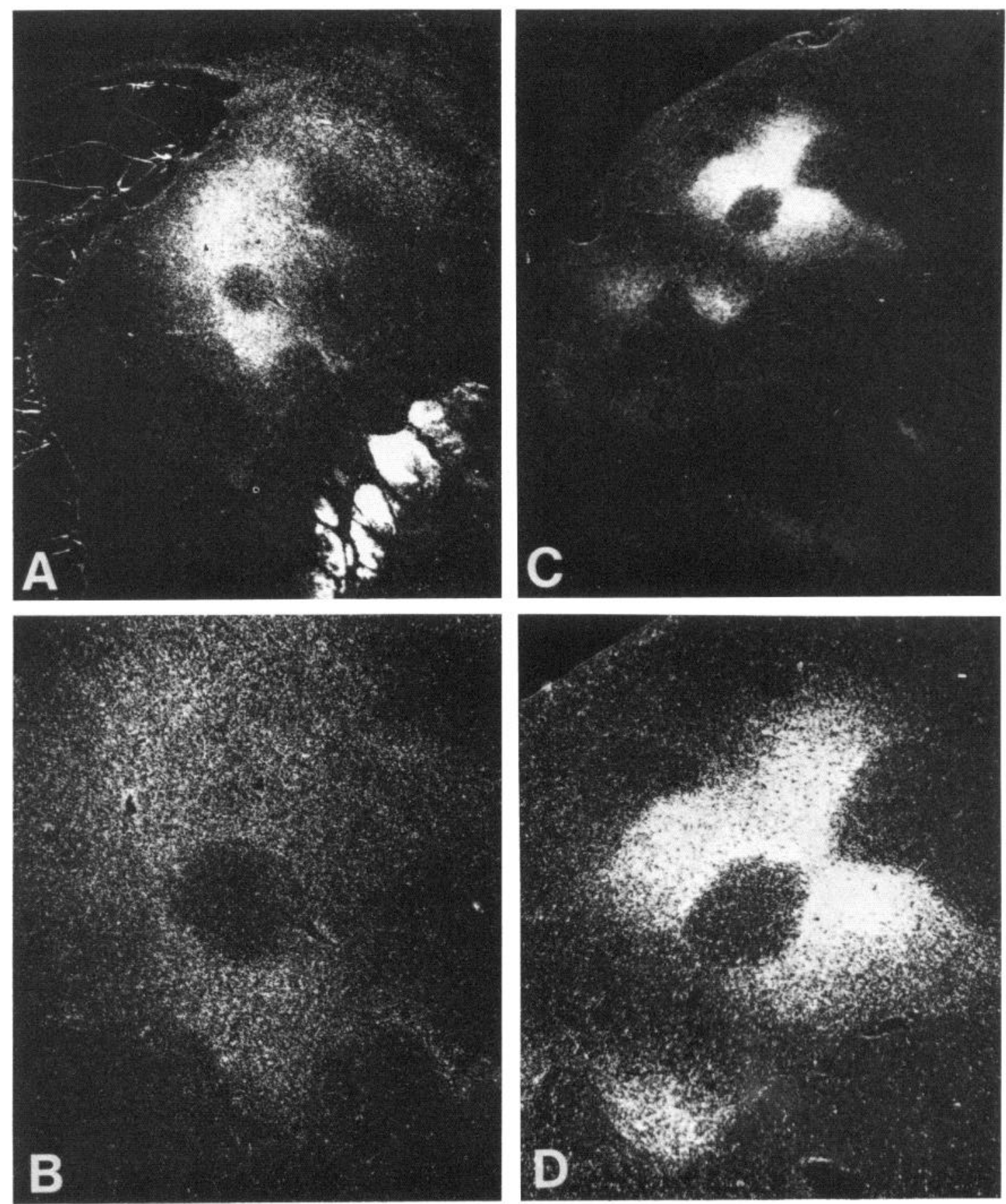

Figure 7. Lower $(\times 22)$ and higher $(\times 50)$ magnification dark-field photographs of corticostriatal projections in a fetus injected at E133 and sacrificed at E134 $(A$ and $B)$ and comparable photographs from a monkey injected postnatally at P4 $(C$ and $D)$. These photographs indicate that the long axis cross-sectional diameter of label-free cores that appear in a fetus 1 month before birth (E134) are comparable in size to the 300- to 600- $\mu \mathrm{m}$ cores that are present in postnatal cases (Bugbee and Goldman-Rakic, 1980). The photographs also indicate that the density of corticostriatal fibers still may be increasing over this period as judged by density of grains in autoradiograms.

of lower grain density gradually emerge among areas of higher grain density. At the beginning of this stage, local differences in the intensity of labeling are blurred, but eventually, these boundaries sharpen up and, finally, around E133, the corticostriatal projection field is perforated by sharply demarcated core territories that are relatively free of prefrontal input. It is important to remember, however, that although the distribution of cortical fibers are arranged as in the adult a full month before birth, neither the cortical terminals nor the striatal neurons are fully mature at this time. For example, synaptogenesis in the neostriatum begins as early as E65 

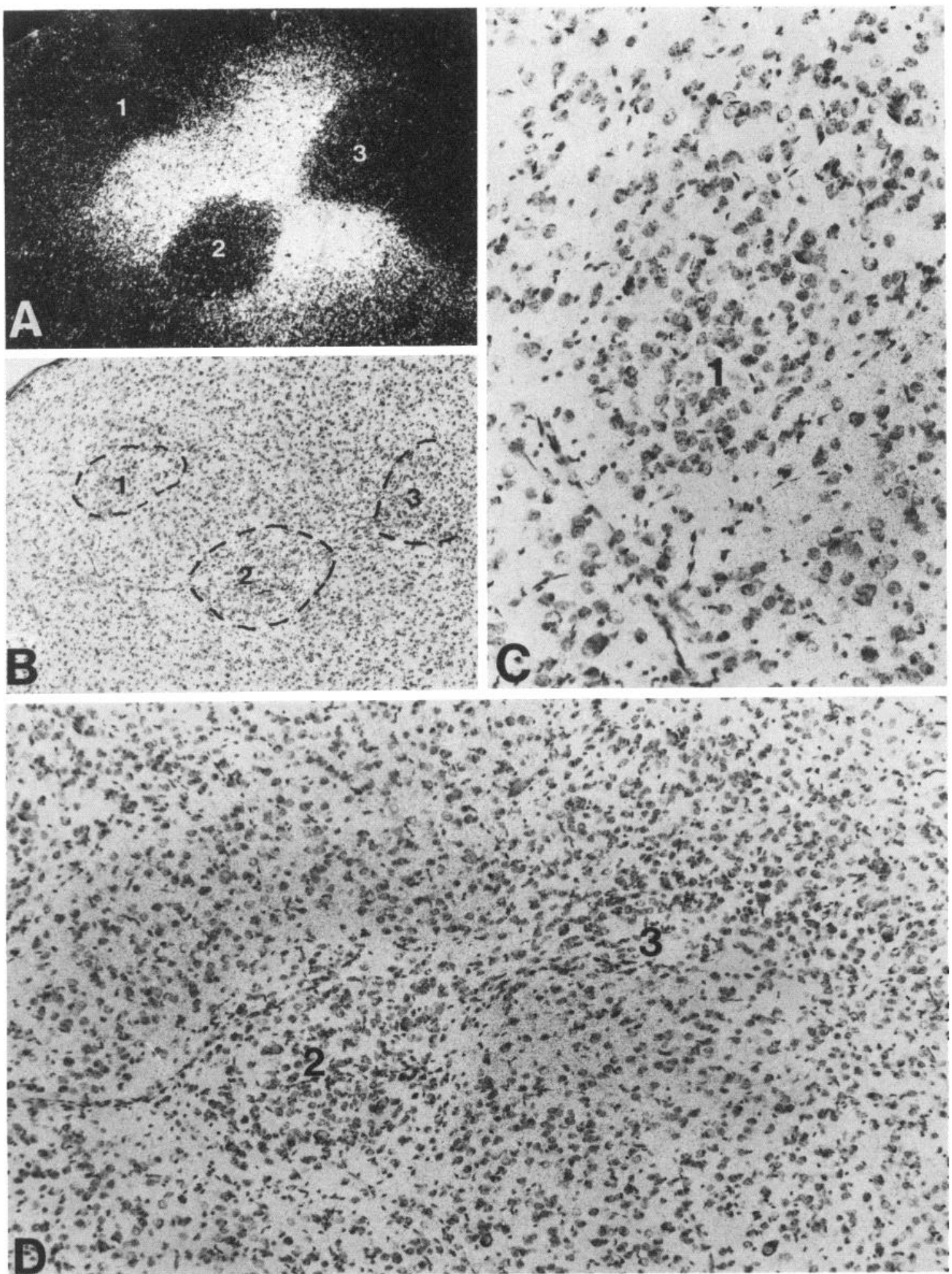

Figure 8. Photographs displaying the relationship between the distribution of corticostriatal input $(A)$ and neostriatal "islands" $(B$ to $D)$ as revealed in a rhesus monkey, the prefrontal cortex of which was injected 1 week prior to sacrifice at P11. $A$, Photograph under dark-field illumination of prefrontostriatal projection to the head of the caudate nucleus with three label-free cores indicated by arabic numbers. Magnification $\times 50$. B, The very same slide as in $A$ photographed under bright-field illumination to expose the three cellular islands that correspond to the label-free cores. $C$ and $D$, Higher magnification bright-field illumination photographs of the same cellular islands $(1,2$, and 3$)$ to display their cytological characteristics as seen in Nisslstained sections: $C, \times 195 ; D, \times 123$. 


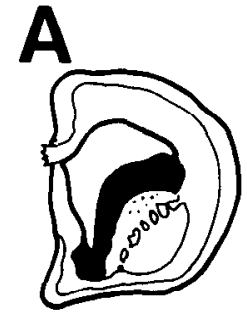

E69-70

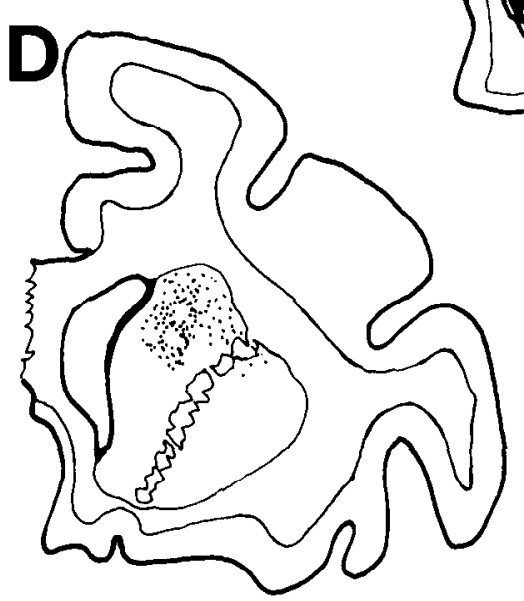

E133-134

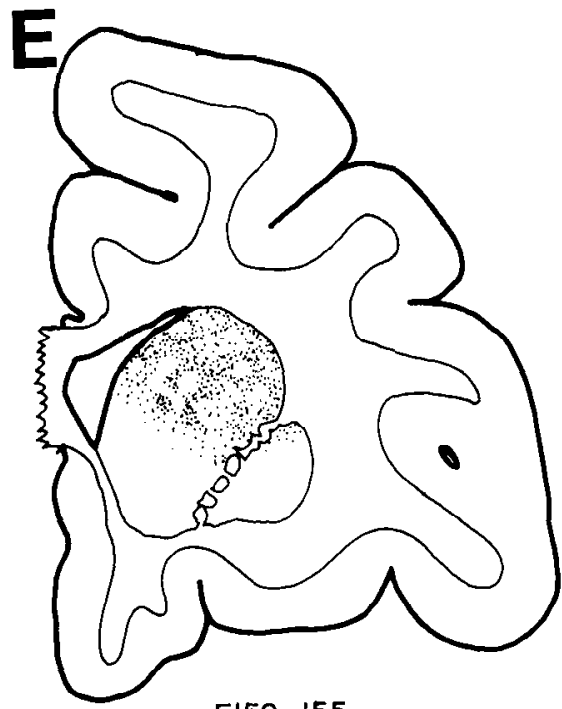

E152- 155

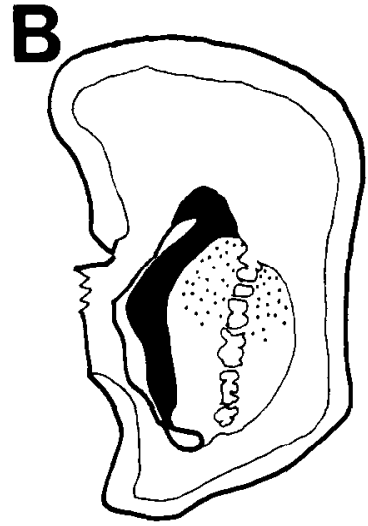

E86-87

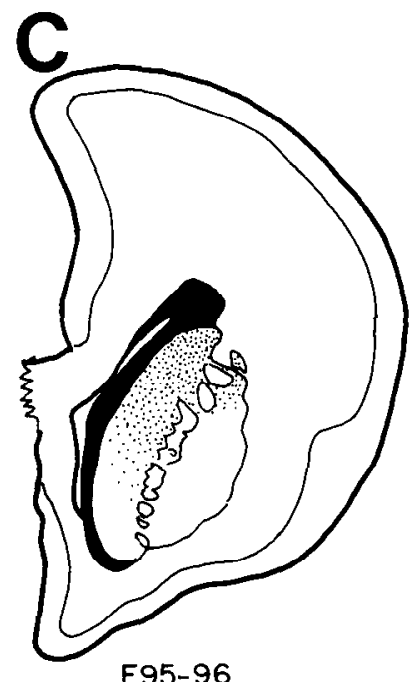

E95-96

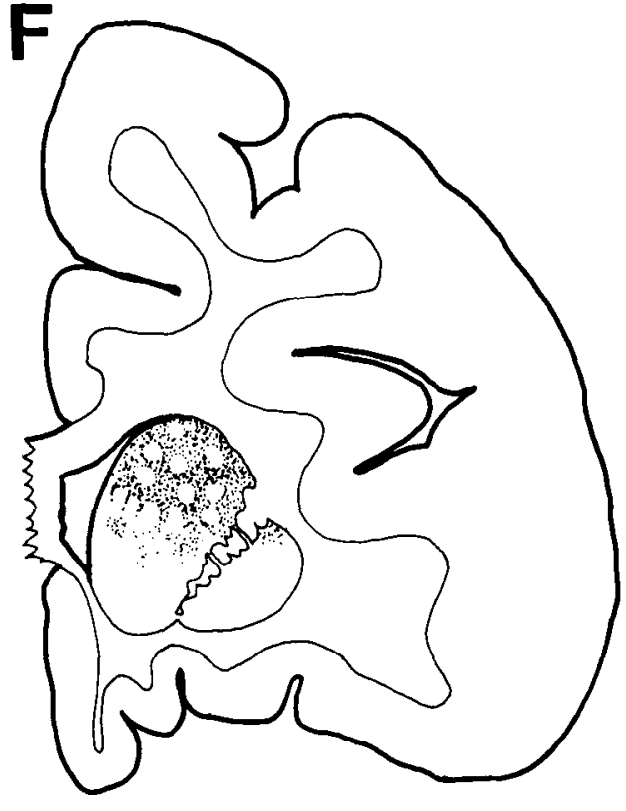

P4-11

Figure 9. Summary semi-diagrammatic illustration of the time and mode of establishment of corticostriatal projections in the developing rhesus monkey. Following the convention used throughout this article, the first number under each drawing refers to the embryonic (E) or postnatal $(\mathrm{P})$ day of $\left[{ }^{3} \mathrm{H}\right]$ proline $/\left[{ }^{3} \mathrm{H}\right]$ leucine injection into the superior bank of the developing principal sulcus; the second number indicates the day of sacrifice. Around E70, a small contingent of prefrontal fibers enters the territory of the developing neostriatum which is still receiving neurons and glia from the proliferative zone of the ganglionic eminence (black area). Between E86 and E96, when all neostriatal neurons have been generated, many more cortical fibers enter the caudate nucleus and putamen. At these ages, as at earlier fetal stages, the corticostriatal input is distributed uniformly throughout the neostriatum. Beginning around E105 (not shown), fenestrations begin to appear in the cortical terminal fields, a process which is completed by E133, 1 month before birth. The pattern of corticostriatal fiber distribution at E133 appears nearly identical to that observed in older fetuses (E152-155) and neonatal (P4-11) monkeys, though synaptogenesis in this structure is far from complete.

(Brand and Rakic, 1979b), but additional synapses continue to form and differentiate through at least the first 16 weeks of postnatal life (DiFiglia et al., 1980). The present autoradiographic and cytoarchitectonic study demonstrates only that, in the rhesus monkey, the basic pattern of connectivity is well established at least 1 month before birth.

The segregation of corticostriatal input was paralleled 
by and correlated with the development of cytoarchitectonic differentiation of the neostriatum. Although traditionally considered to be cellularly homogeneous (Kemp and Powell, 1970), the neostriatum in the adult rhesus monkey has been shown recently to consist of at least two cytoarchitectonically defined cellular compartments (P. S. Goldman-Rakic, submitted for publication). These are (i) cellular islands that appear oval or elliptical in coronal sections and (ii) a relatively homogeneous cellular matrix that surrounds them. These cellular compartments, like certain cytoarchitectonic boundaries in the neocortex, are not easy to detect by superficial examination. However, they become visible upon careful analysis of Nissl-stained sections and with the guidance of autoradiograms from adjacent sections (P. S. Goldman-Rakic, submitted for publication). Of particular relevance for the developmental analysis of the present study is that, during the second third of gestation, corticostriatal fibers do not respect the boundaries between prospective "island" and "matrix" neurons, as fibers spread indiscriminately among the immature neostriatal cells. However, in the last third of gestation, beginning around E105, fiber terminals disappear or retract from the territory that is emerging as a cellular island and become concentrated in the cell-poor annuli and matrix zones that encircle the developing islands. This process of segregation of fiber input and cytoarchitectonic compartmentalization closely resembles the similar process of segregation of input from the two eyes into separate ipsilateral and contralateral laminae of the lateral geniculate body (Rakic, 1976) or into ocular dominance domains of the visual cortex (Rakic, 1976; Hubel et al., 1977). It also recalls segregation of dual fibers systems from overlapping to spatially distinct territories in the olfactory cortex (Price et al., 1976). The present findings underscore the generality of these phenomena by indicating that the principle followed in the formation of sensory systems also may govern the development of nonsensory connections.

The biphasic development of discontinuous corticostriatal terminal fields and cellular compartments within the neostriatum is also reminiscent of other morphological and biochemical changes that have been described in this structure recently. One can only speculate about how the development of cell aggregates and cortical input may be related to the dopamine fiber "islands" that originate in the substantia nigra (Olson et al., 1972; Tennyson et al., 1972) or to dense and light zones of acetylcholinesterase (AChE) activity observed within the neostriatum (Butcher and Hodge, 1976; Graybiel and Ragsdale, 1979; Kostovic-Knezevic et al., 1979). However, it might be suggested that the patches defined by dense $\mathrm{AChE}$ centers and pale AChE perimeters described in the neostriatum of human fetuses and neonates may correspond, respectively, to the cell clusters and fiber annuli described in the present study. This would suggest the possibility that island cells may be cholinergic or may receive cholinergic input, whereas matrix cells presumably receive glutamatergic input from corticostriatal neurons (Kim et al., 1977). It should not be overlooked, however, that, although both dopamine and acetylcholinesterase staining exhibit discontinuities in the neostriatum of immature rodents, carnivores, and in man, sub- sequently, the uneven distributions of these neurotransmitters is overshadowed by a more diffuse pattern of staining (Olson et al., 1972; Butcher and Hodge, 1976; Graybiel and Ragsdale, 1979). If this pattern of change is a progression from specific to diffuse, as it appears to be on the surface, it is opposite to the diffuse-to-patterned transformation that occurs in the corticostriatal terminal fields in monkey. Thus, many questions about the spatial and temporal relationships of the intrinsic and extrinsic fiber systems within the neostriatum still remain to be answered.

Finally, the relationship between cytoarchitectonically defined cell clusters and neurons that are generated and/ or migrate in clusters should be examined. It is important to emphasize that we have no direct evidence that the cellular islands in Nissl-stained sections bear a relationship to the clusters of cells labeled in postnatal monkeys following $\left[{ }^{3} \mathrm{H}\right]$ thymidine injection during the fetal period of neostriatal neurogenesis (Brand and Rakic, 1979a). In fact, an equivalence of these two types of cellular aggregates is unlikely because it would imply that almost all neostriatal neurons are destined to be island cells, and this clearly cannot be the case.

Mechanisms for sorting out of corticostriatal connections. 'Two basic types of cellular mechanisms can be proposed to account for how corticostriatal fibers are transformed from an initially diffuse to a fenestrated pattern in the mature brain. One possibility is a competition between cortical and other inputs for synaptic space in the various cellular compartments of the developing neostriatum. Such rivalry would imply that corticostriatal terminals are displaced to the periphery of neostriatal islands by an influx of competing fibers from another source, such as the thalanus, the substantia nigra, and/or the midbrain raphe complex, which may have higher affinities than corticostriatal input for the "island" cells. Undoubtedly, this readjustment of cortical terminals is aided by translocations of the target cells and dendrites upon which they terminate. Also, if the corticostriatal projections to the islands have already formed some synaptic junctions before E95, as seems likely (Brand and Rakic, 1979b), the competition hypothesis would require that these are later broken. This would not be unprecedented; indeed elimination of synapses seems to be the rule rather than the exception during development (for review, see Purves and Lichtman, 1980; also Changeux and Danchin, 1976).

Unfortunately, very little is known yet about the synaptic terminations of corticostriatal axons in fetal monkeys nor about their relationship to the timing and sequence of arrival of the various other neostriatal afferents during primate development. Based on present knowledge, it is likely that fibers of subcortical origin invade the neostriatum at least as early as, if not earlier than, the corticostriatal input. Neurons of the brain stem (Levitt and Rakic, 1979) and thalamus (Dekker and Rakic, 1980) which project to the neustriatum are already generated in the monkey between E30 and E45 and between E38 and E43, respectively. However, these fibers may not innervate the neostriatum and differentiate their terminal fields until midgestation. Another likely source of fibers involved in competition for synaptic space may come from other nonfrontal cortical areas of the same 
hemisphere. Competition from homotopic neurons of the opposite hemisphere appears to be ruled out by our evidence of their paucity. Whatever their source, other neostriatal inputs must either overlap the corticostriatal projection during the period of gestation when it is distributed diffusely or else develop later in gestation.

Another possible explanation for the formation of the cortical terminal patterns in the neostriatum is the selective elimination of a subpopulation of cortical neurons that initially projected to the neostriatal islands. Cell death has been considered by many to be one of the principal mechanisms for shaping neural centers and establishing appropriate connections (e.g., Cowan, 1973; Clarke and Cowan, 1975; Knyihar et al., 1978). If this is applicable to the formation of corticostriatal terminal fields, cortical cell death must occur in a nonrandom fashion in order to produce "empty" elliptical areas within fiber clusters.

Any mechanism that may be responsible for the developmental transformation from a diffuse to a fenestrated distribution of corticostriatal axon terminals also will have to account for the formation of cellular islands in the neostriatum. The developmental interdependence of cortical and neostriatal neurons needs to be elaborated further in terms of whether cytological changes, such as island formation in the target structure, induce or are induced by segregation of ingrowing axons. Since the neostriatum is becoming well characterized anatomically, histochemically, and biochemically, the formation of compartments in this structure may provide a valuable model for the analysis of mechanisms involved in neuronal specificity in complex systems. Its value as a model is enhanced further by the present evidence that a transformation of corticostriatal input from overlapping and diffuse to a segregated pattern appears to reflect a general rule governing the formation of central neuronal connections-one that applies not only to the ascending radiations of sensory afferent systems but also to the major descending efferent projections of the association neocortex.

\section{References}

Brand, S., and P. Rakic (1979a) Genesis of the primate neostriatum: $\left[{ }^{3} \mathrm{H}\right]$ Thymidine autoradiographic analysis of the time of neuron origin in the rhesus monkey. Neuroscience 4: 767778.

Brand, S., and P. Rakic (1979b) Synaptogenesis in the caudate nucleus of pre- and postnatal rhesus monkeys. Anat. Rec. 193: 490 .

Bugbee, N. M., and P. S. Goldman-Rakic (1980) Compartmentalization of prefrontal projections: Comparisons of cortical columns and striatal islands in old and new world monkeys. Soc. Neurosci. Abstr. 6: 822.

Butcher, L. L., and G. K. Hodge (1976) Postnatal development of acetylcholinesterase in the caudate-putamen nucleus and substantia nigra of rats. Brain Res. 106: 223-240

Changeux, J. .P., and A. Danchin (1976) Selective stabilization of developing synapses as mechanism for the specification of neuronal networks. Nature 264: 705-712.

Clarke, P. G. H., and W. M. Cowan (1975) Ectopic neurons and aberrant connections during neuronal development. Proc. Natl. Arad. Sci. U. S. A. 72: 4455-4458.

Cospito, J. A., M. S. Levine, and A. M. Adinolfi (1980) Organi- zation of developing precruciate corticostriate projections in kittens. Exp. Neurol. 67: 447-452.

Cowan, W. M. (1973) Neuronal death as a regulative mechanism in the control of cell number in the nervous systern. In Development and Aging in the Nervous System, M. Rockstein, ed., pp. 59-86, Academic Press, New York.

Cowan, W. M., D. I. Gottleib, A. E. Hendrickson, J. L. Price, and T. A. Woolsey (1972) The autoradiographic demonstration of axonal connections in the central nervous system. Brain Res. 37: 21-51.

Crane, A., and P. S. Goldman (1979) An improved albumingelatin method for embedding brain tissue. Stain Technol. 54: 71-75

Dekker, J. J., and P. Rakic (1980) Genesis of the neurons in the motor cortex and VA-VL thalamic complex in rhesus monkey. Soc. Neurosci. Abstr. 6: 205.

DiFiglia, M., P. Pasik, and T. Pasik (1980) Early postnatal development of the monkey neostriatum: A Golgi and ultrastructural study. J. Comp. Neurol. 190: 303-331.

Fallon, J. H., and B. T. S. Ziegler (1979) The crossed corticocaudate projection in the rhesus monkey. Neurosci. Lett. 15: 29-32.

Goldman, P. S. (1978) Neuronal plasticity in primate telencephalon: Anomalous crossed cortico-caudate projections induced by prenatal removal of frontal association cortex. Science 202: 768-770.

Goldman, P. S., and T. W. Galkin (1978) Prenatal removal of frontal association cortex in the rhesus monkey: Anatomical and functional consequences in postnatal life. Brain Res. 52: 451-485.

Goldman, P. S., and W. J. H. Nauta (1977) An intricately patterned prefrontocaudate projection in the rhesus monkey. J. Comp. Neurol. 171: 369-386.

Goldman-Rakic, P. S. (1981) Development and plasticity of primate frontal association cortex. In The Cerebral Cortex, F. O. Schmitt, F. G. Worden, and S. D. Dennis, eds., pp. 6997, MIT Press, Cambridge, MA.

Graybiel, A. M., and C. W. Ragsdale (1979) Histochemically distinct compartments in the striatum of human being, monkey, and cat demonstrated by the acetylthiocholinesterase staining method. Proc. Natl. Acad. Sci. U. S. A. 75: 57235726.

Hubel, D. H., T. N. Wiesel, and S. LeVay (1977) Plasticity of ocular dominance columns in monkey striate cortex. Philos. Trans. R. Soc. Lond. (Biol.) 278: 377-409.

Innocenti, G. M., L. Fiore, and R. Caminiti (1977) Exuberant projections into the corpus callosum from the visual cortex of newborn cats. Neurosci. Lett. 4: 237-242.

Jones, E. G., J. D. Coulter, H. Burton, and R. Porter (1977) Cells of origin and terminal distribution of corticostriatal fibers arising in the sensory-motor cortex of monkeys. J. Comp. Neurol. 173: 53-80.

Kemp, J. M., and T. P. S. Powell (1970) The cortico-striate projection in the monkey. Brain 93: 525-546.

Kim, J. -S., R. Hassler, P. Haug, and K. -S. Paik (1977) Effect of frontal cortex ablation on striatal glutamic acid level in rat. Brain Res. 132: 370-374.

Kostovic-Knezevic, Lj., I. Kostovic, J. Krompotic-Nemanic, and Z. Kelovic (1979) Acetylcholinesterase (AChE) staining in the growing telencephalic structures of the human fetus. Verh. Anat. Ges. 73: 667-669.

Knyihar, E., B. Csillik, and P. Rakic (1978) Transient synaptic contacts in the embryonic primate spinal cord. Science 202: 1206-1209.

Künzle, H. (1975) Bilateral projections from precentral motor cortex to the putamen and other parts of the basal ganglia. Brain Res. 88: 195-210.

Künzle, H. (1978) An autoradiographic analysis of efferent 
connections from premotor and adjacent prefrontal regions (area 6 and 9) in Macaca fascicularis. Brain Behav. Evol. 15: $185-234$.

Land, P. W., and R. D. Lund (1979) Development of the rat's uncrossed retinotectal pathway and its relation to plasticity studies. Science 205: 698-700.

Levitt, P., and P. Rakic (1979) Genesis of central monoamine neurons in the rhesus monkey. Soc. Neurosci. Abstr. 5: 341

Olson, L., A. Seiger, and K. Fuxe (1972) Heterogeneity of striatal and limbic dopamine innervation: Highly fluorescent islands in developing and adult rats. Brain Res. 44: 283-288.

Price, J. L., G. F. Moxley, and J. E. Schwob (1976) Development and plasticity of complementary afferent fiber systems to the olfactory cortex. Exp. Brain Res. Suppl. 1: 148-154.

Purves, D., and J. W. Lichtman (1980) Elimination of synapses in the developing nervous system. Science 210: 153-157.

Rakic, P. (1976) Prenatal genesis of connections subserving ocular dominance in the rhesus monkey. Nature 261: 467471.
Shatz, C., and P. Rakic (1981) The genesis of efferent connections from the visual cortex of the fetal rhesus monkey. J. Comp. Neurol. 196: 287-307.

Tanaka, D. T., T. Gorska, and K. Kutkiewicz (1980) Corticostriate projection patterns and synaptic morphology in the puppy caudate nucleus. Exp. Neurol. 70: 98-108.

Taub, E., G. Barro, E. Miller, P. N. Perrella, A. Jakniunas, P. S. Goldman, J. M. Petras, C. C. Darrow, and D. F. Martin (1974) Feasibility of spinal cord or brain surgery in fetal rhesus monkeys. Soc. Neurosci. Abstr. 1: 447.

Tennyson, V. M., R. E. Barrett, G. Cohen, L. Cote, R. Ieikkila, and C. Mytilineou (1972) The developing neostriatum of the rabbit: Correlation of fluorescence histochemistry, electron microscopy, endogenous dopamine levels, and ${ }^{3} \mathrm{H}$-dopamine uptake. Brain Res. 46: 251-285.

Yeterian, E. H., and G. W. Van Hoesen (1978) Cortico-striate projections in the rhesus monkey: The organization of certain cortico-caudate connections. Brain Res. 139: 43-63. 